\title{
Effect of the silane concentration on the selected properties of an experimental microfilled composite resin
}

\author{
Cesar Henrique Zanchi ${ }^{1}$, Fabrício Aulo Ogliari ${ }^{2}$, Ricardo Marques e Silva ${ }^{2}$, Rafael Guerra Lund ${ }^{3}$, \\ Heloisa Har Machado ${ }^{1}$, Carlo Prati ${ }^{4}$, Neftalí Lenin Villarreal Carreño ${ }^{2}$ and Evandro Piva ${ }^{3 *}$ (]
}

${ }^{*}$ Correspondence:
piva@ufpel.edu.br
${ }^{3}$ Department of Operative
Dentistry, School of Dentistry,
Federal University of Pelotas,
457 Gonçalves Chaves Street,
Pelotas, RS $96015-560$, Brazil
Full list of author information
is available at the end of the
article

*Correspondence: piva@ufpel.edu.br

${ }^{3}$ Department of Operative Federal University of Pelotas, 457 Gonçalves Chaves Street, Pelotas, RS 96015-560, Brazil is available at the end of the

\begin{abstract}
The aim of present study was evaluate the effect of different percentages of an organofunctionalized silane monomer as adhesion promoter between barium borosilicate glass fillers and (co)monomer blend in experimental dental composite resin. Gammamethacryloxypropyltrimethoxysilane ( $\gamma$-MPS) was assessed in an experimental luting cement, at the concentrations of $0,1,3,5,7$ and 10 (wt\%). The experimental resin without fillers was used as control group. The flexural strength (FS) and elastic modulus (E) were obtained by mini-flexural test and expressed in $\mathrm{MPa}$ and $\mathrm{GPa}$, respectively. Water sorption (WS) and solubility (SL) were determined based on ISO standard 4049:2000. Kruskal-Wallis and Student-Newman-Keuls test were used for comparisons of FS, E and WS. The comparisons of SL means were performed using one-way ANOVA and Tukey's method ( $a=5 \%$ ). The treatment with $3 \%$ silane revealed statistically higher FS, while the group treated with $1 \%$ silane showed statistically higher $E$ than $3 \%$ silane $(p<0.05)$ and $E$ similar to control. The experimental composite without filler content showed the highest SL $(p<0.05)$ while the control composite showed the highest WS ( $p<0.05$ ). Based on present findings, flexural strength and elastic modulus can sometimes be improved with lower concentrations (1-3\%) rather than higher concentrations (5-7\%) of the silane ( $\gamma$-MPS) used as coupling agent on barium borosilicate glass filler microparticles of the dental composite resin.
\end{abstract}

Keywords: Polymer composites, Mechanical properties, Dental composite, Silane coupling agent, Microfiller

\section{Background}

In spite of recent advances in contemporary dental composites, materials have retained Bowen's basic triad of core structures of an organic phase (monomers blends), inorganic phase (fillers) and coupling agent (organosilane) [1]. Traditionally the adhesion between fillers and the organic matrix in dental composites is promoted by coupling agents through a filler-matrix interphase (FMI). Comparatively, the FMI has the smallest volume in the composite body, but it is a fundamental phase to provide composite resins with satisfactory properties [2-4]. The concentration of the coupling agent used in the treatment of fillers must be adjusted as a means of resist transfer of the stress from the flexible organic matrix to the stiffness of fillers, thereby protecting the FMI against

(C) 2015 Zanchi et al. This article is distributed under the terms of the Creative Commons Attribution 4.0 International License (http:// creativecommons.org/licenses/by/4.0/), which permits unrestricted use, distribution, and reproduction in any medium, provided you give appropriate credit to the original author(s) and the source, provide a link to the Creative Commons license, and indicate if changes were made. 
fracture [5], reducing the degradation processes and reaching satisfactory hydrolytic stability [6].

The effectiveness of treatment with fillers depends on the nature and amount of the coupling agent, and the composition, size and distribution of the fillers. Although titanates and zirconates can be used as coupling agent, organosilanes, such as $\gamma$-methacryl oxypropyltrimethoxysilane ( $\gamma$-MPS), are those most commonly used. In resin composite systems the organo-functional polymerizable group forms a covalent-bond with the polymerizable monomer groups (vinyl groups or $-\mathrm{C}=\mathrm{C}-$ ) during composite polymerization in the dental office. Furthermore, after activation (hydrolysis), the three chemically labile hydrolyzable alkoxy groups, become intermediate silanols $(\mathrm{Si}-\mathrm{OH})$ and condense around the fillers linking to superficial hydroxyls groups and forming covalent bonds (oxane bond formation, $\mathrm{Si}-\mathrm{O}-\mathrm{Si}$ ) [7].

Nowadays, several types and sizes of fillers have been used in dental composites. Micro-sized barium borosilicate glass fillers are widely used in conventional composite resins, resin-based luting cements and resin-modified glass ionomers. These microfillers may also have different dimensions that require adequate amounts of silane for pre-determined surface areas.

The optimal concentration of silane molecules in the siloxane bridge can be investigated using a mechanical test [7]. When the fillers are treated with a higher percentage of silane than is necessary, they may undergo chemical deterioration. In addition, insufficient filler coating increases the composite viscosity, leading to non-uniform filler dispersion with weak bonds to the organic matrix, resulting in a composite with lower mechanical and physical properties [8]. Furthermore, in the oral environment composite resins can absorb water and release chemical compounds. The water sorption and solubility phenomenon are associated with physical changes such as plasticization, swelling and chemical changes such as oxidation and hydrolysis [9]. Thus, the hydrolysis of the silane coupling agent is widely accepted as one of the main causes of composite material degradation $[10,11]$, leading to a shortened service life of dental restorations.

In dentistry, the concentration of ready-to-use silanes varies between 0.5 and $5 \mathrm{vol} \%$ [8]. However, there is no consensus in the literature about the optimal silane percentage weight for microfiller treatment, since there is conflicting information about the adequate arrangement of the silane molecules according to the surface area of the inorganic particle [12]. Therefore, the aim of this study was to investigate whether different concentrations of silane ( $\gamma$-MPTS) in the treatment of microfillers in the experimental composite resins (ECR) would change the properties evaluated. The null hypothesis tested was that different silane contents (wt\%) would not influence the flexural strength, Young's modulus, water sorption and water solubility of the ECRs.

\section{Methods}

\section{Formulation of the experimental composite resin (ECR)}

The experimental resin blends were formulated by mixing the monomers 2,2-bis[4(2-hydroxy-3-methacryloyloxypropyl)phenyl]-propane (BisGMA) and triethylene glycol dimethacrylate (TEGDMA) obtained from Esstech Inc. (Essington, PA, USA), which were used in $60 / 40 \mathrm{wt} \%$ ratio. To make the materials light polymerizable, a binary light polymerizing system formed of 0.4 of camphorquinone (CQ, Esstech) and 0.8 (wt\%) of 
ethyl 4-dimethylaminobenzoate (EDAB, Fluka, Milwalkee, WI, USA) were dissolved in the co-monomers. The reagents were used as received without further purification.

The barium borosilicate glass fillers (average particle size of $4 \mu \mathrm{m}$, Esstech Inc., Essington, PA, USA) were silanized by gamma-methacryloxypropyltrimethoxysilane ( $\gamma$-MPS, Aldrich Chemical Co., Milwalkee, WI, USA) according to the percentage weight of silane: 0, 1, 3, 5, 7 and 10 (wt\%). The fillers were added in an acetone (Labsynth Ltda., Diadema, SP, Brazil) solution containing $\gamma$-MPS and a slurry was formed. The mixture was stored for $24 \mathrm{~h}$ at $37^{\circ} \mathrm{C}$ to assure the complete solvent removal. After storage, the fillers were sieved through a $150 \mu \mathrm{m}$ sieve and kept in a sonication device for $10 \mathrm{~min}$ for de-agglomeration. The sieved fillers were added to the resin blend, $60 \%$ by weight, and mechanically mixed with a motorized mixer (stirring). In order to assure the adequate dispersion of the fillers and decrease air voids, the ECR were ultrasonicated for $1 \mathrm{~h}$. As a control group, monomer content without fillers was used.

\section{Flexural strength and Young's modulus}

Flexural strength (FS) and Young's modulus $(E)$ were obtained by the mini-flexural test. Seventeen bar shaped specimens were made of each experimental ECR, using a metal mold with internal dimensions of $12.0( \pm 0.1) \mathrm{mm}$ length, $2.0( \pm 0.1) \mathrm{mm}$ width and 2.0 $( \pm 0.1) \mathrm{mm}$ thickness. The mold was placed on a glass slide and a Mylar strip and filled with the uncured samples, which were inserted in a single increment. Another Mylar strip was placed on the mold and pressed against it with a glass slide to remove excess material before polymerization. A LED light-curing unit (Radii, SDI, Bayswater, Victoria, Australia) with irradiance of approximately $1000 \mathrm{~mW} / \mathrm{cm}^{2}$ was used. The LED light intensity was measured with a radiometer Model 100 (Demetron Research Corp., Danbury, CT, USA) before use. The experimental resin luting cements were light polymerized for $40 \mathrm{~s}$ in two consecutive points, producing a partial overlapping. The excess material in the corner was carefully removed with a scalpel blade and the specimens were stored at room temperature for 7 days. After storage, the samples were submitted to a three-point bending test using a universal testing machine (DL-500, Emic, São José dos Pinhais, Brazil) at a crosshead speed of $1 \mathrm{~mm} / \mathrm{min}$. The maximum load was obtained in N. and the flexural strength (FS) was calculated in MPa using the following:

$$
F S=3 F L /\left(2 B H^{2}\right)
$$

where $F$ is the maximum load (in Newtons); $L$ is the distance between the supports (in millimeters); $B$ is the width of the specimen (in millimeters) and $H$, the height (in millimeters).

The Young's modulus $(E)$ was determined in GPa, as follow;

$$
E=F L^{3} / 4 B H^{3} d
$$

where $F$ is the maximum load; $L$ is the distance between the supports; $B$ is the width of the specimen, $H$ is the height of the specimen, and $d$ is the deflexion (in millimeters) corresponding to the load $F$. 
Water sorption and solubility

Water sorption and solubility were determined based on the ISO 4049:2000 specification [13] except for the disc dimensions and number of specimens. Seven disc shaped specimens were obtained from an aluminum mold between two glass slides covered with polyethylene film. Specimens were $6.0 \mathrm{~mm}$ in diameter and $1.0 \mathrm{~mm}$ thick. After removing the glass slide, the specimens were irradiated with a LED light-curing unit (Radii, SDI, Bayswater, VIC, Australia), with exposure of $40 \mathrm{~s}$ on both top and bottom surfaces. Immediately after polymerization, the specimens were placed in a desiccator containing freshly dried silica gel and calcium chloride. After $24 \mathrm{~h}$, the samples were removed, stored in a desiccator at $23{ }^{\circ} \mathrm{C}$ for $1 \mathrm{~h}$ and weighed on a balance with a precision of $0.01 \mathrm{mg}$ (AUW220D, Shimadzu, Kyoto, Japan). This cycle was repeated until a constant mass $\left(m_{1}\right)$ was obtained. Thickness and diameter of the specimens were randomly measured at five points using a digital caliper, rounded to the nearest $0.01 \mathrm{~mm}$, and these measurements were used to calculate the volume $(\mathrm{V})$ of each specimen (in $\mathrm{mm}^{3}$ ). The discs were immersed in distilled water at $37{ }^{\circ} \mathrm{C}$ for 7 days, then removed, blotted dry and weighed $\left(m_{2}\right)$. After weighing, the specimens were dried again inside a desiccator and weighed daily to record a third constant mass $\left(m_{3}\right)$ as previously described. For each disc, the data of water sorption (WS) and solubility (SL) were calculated using the following formulae:

$$
\mathrm{WS}=\left(m_{2}-m_{3}\right) / \mathrm{V} \quad \mathrm{SL}=\left(m_{1}-m_{3}\right) / \mathrm{V}
$$

\section{Statistical analysis}

The flexural strength, Young's modulus and water sorption data were submitted to Kruskal-Wallis and Student-Newman-Keuls test for multiple comparisons. Data for solubility was submitted to one-way ANOVA and the Turkey test for multiple comparisons between groups. The level of significance was set at $\mathrm{p}<0.05$.

\section{Scanning electron microscopy MEV}

The materials were dissolved in $\sim 20 \mathrm{~mL}$ of acetone solution (Vetec, Rio de Janeiro, Brazil), the suspension was sonicated for $25 \mathrm{~min}$ and pipetted onto silicon wafers. Samples were mounted on stubs and sputter coated with gold before being analyzed by scanning electron microscopy (SEM) at 15 kV (SSX-550; Shimadzu, Tokyo, Japan).

\section{Results and discussion}

Flexural strength, Young's modulus, water sorption and solubility mean values of the ECR are shown in Table 1 . The statistically highest FS was obtained when the microfillers were treated with $3 \%$ silane $(\mathrm{p}<0.05)$. The micro-filler treatments with $1,5,7$ and $10 \%$ silane concentrations resulted in ECR with a similar behavior and presented higher FS than the unfilled resin blend. The addition of micro-fillers without previous silanization showed the statistically lowest FS $(\mathrm{p}<0.05)$. In comparison with the micro-filled ECR, the unfilled resin blend presented the lowest $E(\mathrm{p}<0.05)$. When fillers without silanization, or $1 \%$ presalinized fillers were added to the resin blend the $E$ obtained was statistically higher than it was in the other ECRs. In addition, the FS and $E$ could not be improved when silane concentrations higher than $5 \%$ were used. 
Table 1 Means and standard deviation (ISD) of flexural strength, Young's modulus, water sorption and solubility

\begin{tabular}{|c|c|c|c|c|}
\hline Groups & $\begin{array}{l}\text { Flexural strength } \\
\text { (MPa) }\end{array}$ & $\begin{array}{l}\text { Young's modulus } \\
(\mathrm{GPa})\end{array}$ & $\begin{array}{l}\text { Water sorption } \\
\left(\mu \mathrm{g} / \mathrm{mm}^{3}\right)\end{array}$ & $\begin{array}{l}\text { Solubility } \\
\left(\mu \mathrm{g} / \mathrm{mm}^{3}\right)\end{array}$ \\
\hline Absence of filler & $83.4(5.3)^{C}$ & $8.7(0.9)^{\mathrm{E}}$ & $48.1(1.8)^{B}$ & $7.06(1.6)^{A}$ \\
\hline Particles no-silaned (control) & $71.0(11.3)^{\mathrm{D}}$ & $28.0(4.7)^{\mathrm{A}}$ & $58.4(3.4)^{\mathrm{A}}$ & $1.34(0.7)^{\mathrm{B}}$ \\
\hline $1 \%$ silane & $124.7(20.3)^{\mathrm{B}}$ & $27.3(2.9)^{\mathrm{A}}$ & $30.7(2.1)^{\mathrm{D}}$ & $0.50(1.4)^{B}$ \\
\hline $3 \%$ silane & $147.5(24.8)^{\mathrm{A}}$ & $25.5(3.4)^{\mathrm{B}}$ & $33.1(1.5)^{C}$ & $0.35(1.3)^{B}$ \\
\hline $5 \%$ silane & $136.7(15.7)^{\mathrm{B}}$ & $22.7(2.9)^{C}$ & $31.8(2.2)^{\mathrm{D}}$ & $0.19(0.8)^{B}$ \\
\hline $7 \%$ silane & $129.4(13.7)^{\mathrm{B}}$ & $21.2(1.5)^{\mathrm{D}}$ & $29.6(1.2)^{\mathrm{D}}$ & $0.34(0.4)^{B}$ \\
\hline $10 \%$ silane & $125.6(16.8)^{\mathrm{B}}$ & $21.1(3.6)^{\mathrm{D}}$ & $31.5(2.4)^{\mathrm{D}}$ & $0.98(0.7)^{\mathrm{B}}$ \\
\hline
\end{tabular}

Different letters in columns represent statistically significant differences between groups $(p<0.05)$

The combination of monomers and glass filler components favored the development of many composite dental materials suitable for different clinical applications such as conventional restorative composite resin, resin-based luting cements, filled adhesive systems and resin-modified glass ionomers. The integration and adhesion between inorganic fillers and the organic matrix is a key factor in achieving good physical-mechanical properties and decrease in hydrolytic degradation $[8,14]$.

To be effective, the amount of silane absorbed by the filler surface must be optimal [15]. Incomplete silane coating or thicker layers leads to inadequate bonding with the organic matrix and consequently a reduction in the mechanical properties [16, 17]. According to findings of present study, it was demonstrated that the percentage of $\gamma$-MPS used in the micro-filler silanization can significantly influence some of the properties evaluated. Thus, the null hypothesis was rejected. The treatment with $3 \%$ of silane $(\gamma$-MPS) on barium borosilicate glass fillers presented the highest flexural strength, while it showed lower Young's modulus than the group treated with $1 \%$ silane coupling agent.

These results can be explained by the formation of an adequate covering layer of the microfillers producing an interfacial bond with continuous stress distribution between fillers and matrix. The optimal silanization results in a monomolecular coverage on a silica surface [16]. In this case, the carbonyl functionality has a hydrogen bonding interaction with surface silanols and the hydrolyzed trialkoxy has covalent surface linkages [7]. With the appropriate coupling agent, fracture of the ECR will not occur at the interface, but along the resin matrix [12]. It was demonstrated that the functional silane treated microfiller $(\gamma$-MPS) presents a positive effect on wear resistance, mainly when a high filler concentration (up to $30 \%$ by vol) is used [12]. On the other hand, the flexural strength of the particles no-silaned group showed the lowest mean values. When fillers are added to the resin blend, without previous silane treatment allowing for bonding, these particles will act as internal defects, reducing the material strength.

In the groups treated with high amounts of $\gamma$-MPS (7 and $10 \%)$, although this had no significant effect on the flexural strength, a decrease in Young's modulus (E) was observed when compared with the groups treated with 1, $3 \%$ and control group. The Young's modulus is a mechanical property that describes the relative rigidity of the materials. The decreases in E observed suggest the loss of the ECR ability to relieve the excess of energy during elastic deformation. The Young's modulus is particularly important as 
regards composite resins indicated for use in posterior restorations, since composite resins can undergo excessive deformation and are more prone to catastrophic failures [18]. On the other hand in post-restored teeth, Young's modulus is not an important factor related to failure of the material [19] or endodontically treated teeth restored with composite resin core [20]. Moreover, Young's modulus represents overall features, not only those of silanization but also as regards the range of particle size, volumetric filler fraction and effectiveness of dispersion, as well as the technological processes used by manufacturers. Indeed these parameters are related to long lasting clinical evaluations of composite resins [21]. Moreover, the use of high silane concentration can be used as an approach to decrease viscosity in composite resins [15] formulations intending to improve handling in clinical use, however this approach should be considered carefully since it can impairs selected properties.

When excessive amounts of silane are used in the treatment of fillers, a second layer is formed above the covalent linked silane layer, and prevents the direct formation of a bridge from the inorganic filler to the organic matrix, which weakens the composite resin [7]. Although E can serve as a measure of the debonded filler particle fraction, it is strongly dependent on both the filler concentration and particle size [22]. Concentration and particle size were controlled in present study by using the experimental formulation with similar particle size range confirmed by scanning electron microscopy (average particle size of $4 \mu \mathrm{m}$ ). Since different commercial products are tested and most composites are classified as hybrid due to the use of different sized glass particles, it is difficult to address the effect of different sinalization protocols when making comparisons of materials from different manufacturers.

The approach of filler silanization significantly decreases the WS patterns, when compared with absence of filler and Control group $(\mathrm{p}<0.05)$. No-silaned micro-fillers group showed statistically higher WS mean $(\mathrm{p}<0.05)$. Regarding SL, there was not significant differences among the micro-fillers silaned or no-silaned particles. However, the unfilled resin group present SL mean statistically higher than the micro-filled ECR $(\mathrm{p}<0.05)$. The micromorphology of barium glass particles used in present study are shown in Fig. 1.

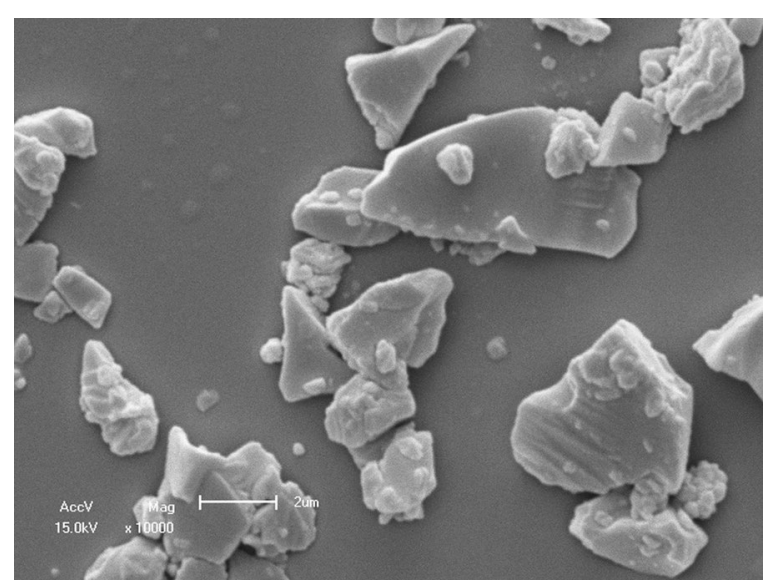

Fig. 1 Scanning electron microscopy image of untreated barium borosilicate glass fillers used in present study 
Although polymer networks are considered largely insoluble structures, with relatively high chemical and thermal stability, in the oral environment composite resins may absorb water and chemicals from the environment and release components into its surroundings. The water uptake (sorption phenomena) results in physical alterations, such as swelling and plasticization, and favors the hydrolytic degradation of polymers by chain scission, functional group cleavage by acids and oxidation [23]. In this study, the without silane ECR group, in which the microfillers were added without previous silane treatment, presented the highest water sorption mean (Table 1). This fact is the result of the strong attraction between the nude fillers and water molecules that penetrated into the polymer network. The free hydroxyls groups on the filler surface attract water forming hydrogen bonds (adsorption). On a lower scale, it is also possible that the water could cause the erosion of the filler particle surfaces [24]. In this study, when the micro-fillers were previously silanized a significant reduction in this phenomenon was observed, except for the $3 \%$ silane group, which showed higher water sorption. Probably this percentage resulted in optimal microfiller coverage, forming a parallel monomolecular silane layer, and consequently reducing the number of free hydroxyls [16].

In comparison with the silanized ERCs, the pure organic polymer (absence of filler group) demonstrated the highest degree of water sorption. Presumably, it is expected that pure resins present higher water sorption values than filled resins, once the inorganic fillers are practically inert and decrease the total volume of the organic matrix. Furthermore, this resin polymer presented the highest mean solubility value, while the filled ECRs showed a reduced loss of mass, with similar performance. The resistance to hydrolytic degradation with suitable silanization approach is supported for other studies [15]. The water uptake followed the Fickian diffusion kinetics [25] and reached approximately $1.0-1.6 \%$ by volume [26]. During this process, water molecules diffuse into the polymer network reducing interchain interactions, such as entanglements and secondary bonding, creating a volumetric expansion called swelling. Moreover, polymer plasticization allows the elution of unreacted monomers, polymerization promoters, oligomers and their by-products, such as methacrylic acid and formaldehyde from the network, and may constitute a biological concern [23]. However microfillers are too large to diffuse through the polymer network, and are practically inert in water, which may explain the similar behavior between the filled ERCs.

In addition to optimized sinalization coating, the de-agglomeration and dispersion of fillers in resin are important factors that may reflects on physical-mechanical properties in composite resin [27]. In the present study $70 \%$ ethanol was used to promote deagglomeration, and this protocol has been used in other studies [7, 27]. Ultrassonication and the supercritical coating [27] method also can be considered especially when there are significant amounts of nanoparticles present in the composition of the inorganic phase of composite resins.

\section{Conclusions}

Based on the results obtained in this study it can be concluded that the $3 \%$ (by weight) of the silane ( $\gamma$-MPS) was the most adequate percentage for the treatment of the barium borosilicate glass fillers with an average particle size of $4 \mu \mathrm{m}$, because it promoted improvement in the flexural strength of the experimental composite resin tested. 


\section{Authors' contributions}

$\mathrm{CHZ}$, was the main researcher who leaded the materials and methods followed by his student HHM responsible for mechanical tests, water sorption and solubility method. FAO and RGL, participated as supervisors of chemical protocol for optimization and use of gamma-methacryloxypropyltrimethoxysilane ( $($-MPS) in silanization method used in present study. NLVC and RMS, contributed regarding characterization of scanning electron microscopy and characterization of microparticles. CP (Italy) and EP (Brazil) were the responsible for study design, financial support, statistics and final review of this project. This project was carried out as an activity of sandwich program of $\mathrm{CHZ}$ (as Ph.D. student of PostGraduate program) at University of Bologna (Italy) under supervision of CP. All authors read and approved the final manuscript.

\section{Author details}

${ }^{1}$ School of Dentistry, Federal University of Pelotas, 457 Gonçalves Chaves Street, Pelotas, RS 96015-560, Brazil. ${ }^{2}$ Materials Engineering School, Technology Development Center, Federal University of Pelotas, 809 Felix da Cunha Street, Pelotas, RS 96010-000, Brazil. ${ }^{3}$ Department of Operative Dentistry, School of Dentistry, Federal University of Pelotas, 457 Gonçalves Chaves Street, Pelotas, RS 96015-560, Brazil. ${ }^{4}$ Department of Endodontics, School of Dentistry, University of Bologna, Via San Vitale 59, Bologna, Italy.

\section{Acknowledgements}

The authors are grateful to the National Council of Research (CNPq/Brazil) for the scholarship and research support (\# 308087/2011-9) and to Esstech Inc. for the donation of reagents.

\section{Competing interests}

The authors declare that they have no competing interests.

Received: 22 November 2015 Accepted: 13 December 2015

Published online: 22 December 2015

\section{References}

1. Yokota MJ, Gilchrist EM, Cox DS, Hall IK, Gagliani J, Giesy RK. Continuous processing/in-situ curing of incrementally applied resin matrix composite materials. US Patent \# 52661391993.

2. Wilson KS, Zhang K, Antonucci JM. Systematic variation of interfacial phase reactivity in dental nanocomposites. Biomaterials. 2005;26:5095-103. doi:10.1016/j.biomaterials.2005.01.008.

3. Wilson KS, Antonucci JM. Interphase structure-property relationships in thermoset dimethacrylate nanocomposites. Dent Mater. 2006;22:995-1001. doi:10.1016/j.dental.2005.11.022.

4. Wilson KS, Allen AJ, Washburn NR, Antonucci JM. Interphase effects in dental nanocomposites investigated by small-angle neutron scattering. J Biomed Mater Res A. 2007:81:113-23. doi:10.1002/jbm.a.30975.

5. Calais JG, Soderholm KJ. Influence of filler type and water exposure on flexural strength of experimental composite resins. J Dent Res. 1988;67:836-40.

6. Soderholm KJ. Degradation of glass filler in experimental composites. J Dent Res. 1981;60:1867-75.

7. Sideridou ID, Karabela MM. Effect of the amount of 3-methacyloxypropyltrimethoxysilane coupling agent on physical properties of dental resin nanocomposites. Dent Mater. 2009;25:1315-24. doi:10.1016/j.dental.2009.03.016.

8. Matinlinna J, Ozcan M, Lassila L, Kalk W, Vallittu P. Effect of the cross-linking silane concentration in a novel silane system on bonding resin-composite cement. Acta Odontol Scand. 2008;66:250-5. doi:10.1080/00016350802247131.

9. Ferracane JL. Hygroscopic and hydrolytic effects in dental polymer networks. Dent Mater. 2006;22:211-22. doi:10.1016/j.dental.2005.05.005.

10. Ferracane $J \mathrm{~L}$, Marker VA. Solvent degradation and reduced fracture toughness in aged composites. J Dent Res. 1992;71:13-9.

11. Soderholm KJ, Zigan M, Ragan M, Fischlschweiger W, Bergman M. Hydrolytic degradation of dental composites. J Dent Res. 1984;63:1248-54.

12. Lim BS, Ferracane JL, Condon JR, Adey JD. Effect of filler fraction and filler surface treatment on wear of microfilled composites. Dent Mater. 2002;18:1-11.

13. Moreira FCL, Filho NRA, Souza JB, Lopes LG. Sorption, solubility and residual monomers of a dental adhesive cured by different light-curing units. Braz Dent J. 2010;21:432-8.

14. Karabela MM, Sideridou ID. Effect of the structure of silane coupling agent on sorption characteristics of solvents by dental resin-nanocomposites. Dent Mater. 2008;24:1631-9. doi:10.1016/j.dental.2008.02.021.

15. Karmaker A, Prasad A, Sarkar NK. Characterization of adsorbed silane on fillers used in dental composite restoratives and its effect on composite properties. J Mater Sci Mater Med. 2007;18:1157-62. doi:10.1007/s10856-007-0145-y.

16. Soderholm KJ, Shang SW. Molecular orientation of silane at the surface of colloidal silica. J Dent Res. 1993;72:1050-4.

17. Nihei T, Dabanoglu A, Teranaka T, Kurata S, Ohashi K, Kondo Y, Yoshino N, Hickel R, Kunzelmann KH. Three-body-wear resistance of the experimental composites containing filler treated with hydrophobic silane coupling agents. Dent Mater. 2008;24:760-4. doi:10.1016/j.dental.2007.09.001.

18. Willems G, Lambrechts P, Braem M, Celis JP, Vanherle G. A classification of dental composites according to their morphological and mechanical characteristics. Dent Mater. 1992;8:310-9.

19. Stewardson DA, Shortall AC, Marquis PM. The effect of the elastic modulus of endodontic posts on static load failure. Int Endod J. 2011;44:458-68. doi:10.1111/j.1365-2591.2011.01851.x.

20. Kumagae N, Komada W, Fukui Y, Okada D, Takahashi H, Yoshida K, Miura H. Influence of the flexural modulus of prefabricated and experimental posts on the fracture strength and failure mode of composite resin cores. Dent Mater $J$. 2012;31:113-9. 
21. Rodolpho DR, Donassollo PA, Cenci TAMS, Loguercio AD, Moraes RR, Bronkhorst EM, Opdam NJ, Demarco FF. 22-year clinical evaluation of the performance of two posterior composites with different filler characteristics. Dent Mater. 2011;27:955-63. Doi:10.1016/j.dental.2011.06.001.

22. Babich VF, Lipatov YS, Todosijchuk TT. Filler debonding in particulate-filled composites. J Adhes. 1996:55:317-27.

23. Ferracane JL. Developing a more complete understanding of stresses produced in dental composites during polymerization. Dent Mater. 2005;21:36-42. doi:10.1016/j.dental.2004.10.004.

24. Milleding P, Karlsson S, Nyborg L. On the surface elemental composition of non-corroded and corroded dental ceramic materials in vitro. J Mater Sci Mater Med. 2003;14:557-66.

25. Sideridou I, Achilias DS, Spyroudi C, Karabela M. Water sorption characteristics of light-cured dental resins and composites based on Bis-EMA/PCDMA. Biomaterials. 2004;25:367-76.

26. Ferracane JL. Water sorption and solubility of experimental dental composites. Abstracts of papers of the American Chemical Society, 214, 142-POLY; 1997.

27. Stojanović DB, Brajović L, Orlović A, Dramlić D, Radmilović V, Uskoković PS, Aleksić R. Transparent PMMA/silica nanocomposites containing silica nanoparticles coating under supercritical conditions. Prog Org Coat. 2013;76:626-31.

\section{Submit your manuscript to a SpringerOpen ${ }^{\circ}$} journal and benefit from:

- Convenient online submission

- Rigorous peer review

- Immediate publication on acceptance

- Open access: articles freely available online

- High visibility within the field

- Retaining the copyright to your article

Submit your next manuscript at $\boldsymbol{s p r i n g e r o p e n . c o m ~}$ 